\title{
Lieber Leser,
}

am Anfang dieses Buches muss (leider) eine Entschuldigung stehen. Der vorliegende Band war, zusammen mit einer englischen Ausgabe, in Japan bereits gedruckt, als wir feststellen mussten, dass auf Seite 179 versehentlich reits gedruckt, als wir feststellen mussten, dass auf Seite 179 versehentlich die entsprechende Seite der englischen Ausgabe in unser Buch geraten war. klebt werden. Wir bitten um Ihr Verständnis und um Ihre Nachsicht.

\section{Ihr Birkhäuser Verlag}




\section{BILDQUELLEN}

Der Verfasser mochte den nachfolgend Genannten für die Großzügigkeit danken, mit der sie Photographien für dieses Buch zur Verfügung gestellt haben. Das Copyright (C) gehört bei allen Photographien den genannten Personen oder Institutionen, die Photographien dürfen nicht ohne ihre Erlaubnis reproduziert werden

\begin{tabular}{|c|c|}
\hline Photo & Objekt \\
\hline $\begin{array}{l}\text { Fronti- } \\
\text { spiz }\end{array}$ & Galaxie NGC 6744 \\
\hline 1 & Die Sonne \\
\hline 2 & Milchstraße \\
\hline 3 & Kohlensacknebel \\
\hline 4 & Kegelnebel \\
\hline 5 & Pferdekopfnebel \\
\hline 6 & Orionnebel \\
\hline 7 & Trapezium \\
\hline 8 & Adlernebel \\
\hline 9 & Rosettanebel \\
\hline 10 & Rosettanebel (Zentralbereich) \\
\hline 11 & Eta Carinae-Nebel \\
\hline
\end{tabular}

12 Eta Carinae-Nebe

(Zentralbereich)

13 Trifid- und Lagunennebe

14 Lagunennebel

15 Trifidnebel

16 Sternhaufen NGC 3293

17 Sternhaufen der Plejaden

18 Kugelsternhaufen NGC2808

19 Kugelsternhaufen $\mathrm{M} 13$

20 Kugelsternhaufen $M 3$

21 Kugelsternhaufen M 15

22 Kugelsternhaufen Omega

$$
\text { Centauri }
$$

23 Kugelsternhaufen M5

24 Kugelsternhaufen 47 Tucana

$25 \quad$ Kugelsternhaufen NGC 6522 und NGC 6528

26 Intergalaktischer

Kugelsternhaufen NGC2419

Planetarischer Nebel M 27

Planetarischer Nebel M57

Schleiernebel (Gesamtansicht)

\section{Quelle}

Raymond J. Talbot, Jr., Reginald

$J$. Dufour, und Eric B. Jensen,

Rice University

United States Naval Research

Laboratory

Mssrs. Brodkorb, Rinm, und

Rusche, Astrophoto Laboratory

Harvard College Observatory

Hale Observatories

Royal Observatory, Edinburgh

Royal Observatory, Edinburgh

Lick Observatory

Hale Observatories

Hale Observatories

Kitt Peak National Observatory

Association of Universities for

Research in Astronomy, Inc.

Cerro Tololo Inter-American

Observatory

Association of Universities for

Research in Astronomy, Inc.

Cerro Tololo Inter-American

Observatory

Hale Observatories

Association of Universities for

Research in Astronomy, Inc.,

Kitt Peak National Observatory

David Malin, Anglo-Australian

Telescope Board

David Malin, Anglo-Australian

Telescope Board

Hale Observatories

Mt. Stromlo und Siding Spring

Observatories, Australian

National Observatory

United States Naval Observatory

Hale Observatories

Kitt Peak National Observatory

Cerro Tololo Inter-American

Observatory

Kitt Peak National Observatory

Cerro Tololo Inter-American

Observatory

Kitt Peak National Observatory

Observatories

Hale Observatories

Hale Observatories

\section{Rene Racine, Hale}

Hale Observatories
Photo Objekt

39

M32

M33

M 101

M64

M 104

M83
Schleiernebel (Ausschnitt) Krebsnebel-Überlagerung

Krebsnebel

Milchstraße im Schwan

Milchstraße im Schützen

Milchstraßen-Mosaik

Große Magellansche Wolke

Die Magellanschen Wolken Kleine Magellansche Wolke Kleine Magellansche Wolke

Galaxie M31

M31 (Zentralgebiet)

Galaxie M31 (Zentralgebiet)

M31 (in Kernnähe)

M31 (äußerer Arm)

M31 (Kern)

NGC 205

NGC 147

NGC 185

M31 (Radiokarte)

Bildhauer Zwerg-Kugel-Galaxie

NGC7331

Galaxien, Formaltypen

NGC2841

NGC 2613

NGC 3623

NGC 4565

NGC 3992

NGC 4541

NGC 1360

NGC 4650

NGC 4548
Quelle

Kitt Peak National Observatory Guido Münch und Walter Baade Hale Observatories

Lick Observatory

National Geographic-Palomar

Observatory Sky Survey

National Geographic-Palomar

Observatory Sky Survey

Mt. Stromlo und Siding Springs

Observatories, Australian

National University

Raymond J. Talbot, Jr., Reginald

J. Dufour, und Eric B. Jensen,

Rice University

Harvard College Observatory

Royal Observatory, Edinburgh

Raymond J. Talbot, Jr., Reginald

$J$. Dufour, und Eric B. Jensen,

Rice University

Hale Observatories

Association of Universities for

Research in Astronomy, Inc.,

Kitt Peak National Observatory

Hale Observatories

Hale Observatories

Hale Observatories

Lick Observatory

Kitt Peak National Observatory

Hale Observatories

Hale Observatories

Lick Observatory

Elly M. Berkhuijsen,

Max-Planck-Institut für

Radioastronomie

Hale Observatories

European Southern

Observatory

Hale Observatories

Hale Observatories

Hale Observatories

Hale Observatories

Allan Sandage, Hale

Observatories

Hale Observatories

United States Naval Observatory

Steven Strom, Kitt Peak National

Observatory

Lick Observatory

Lick Observatory

Steven Strom, Kitt Peak National

Observatory

European Southern

Observatory

Laird A. Thompson, Kitt Peak

National Observatory

Laird A. Thompson, Kitt Peak

National Observatory

Raymond J. Talbot, Jr., Reginald

$J$. Dufour, und Eric B. Jensen,

Rice University 


\begin{tabular}{|c|c|}
\hline Photo & Objekt \\
\hline $\begin{array}{l}75 \\
76\end{array}$ & $\begin{array}{l}\text { M83 (Negativabzug) } \\
\text { M83 (Radiokarte) }\end{array}$ \\
\hline 77 & NGC 4477 \\
\hline $\begin{array}{l}78 \\
79 \\
80 \\
81 \\
82 \\
83 \\
84\end{array}$ & $\begin{array}{l}\text { M84 } \\
\text { M49 } \\
\text { Carina-Zwerggalaxie } \\
\text { NGC } 3077 \\
\text { Zwerggalaxie im Sextanten } \\
\text { NGC5364 } \\
\text { NGC } 6744\end{array}$ \\
\hline $\begin{array}{l}85 \\
86 \\
87 \\
88 \\
89,90 \\
91 \\
92 \\
93 \\
94\end{array}$ & $\begin{array}{l}\text { M74 } \\
\text { NGC } 2683 \\
\text { NGC } 5907 \\
\text { Pluto und NGC } 5248 \\
\text { NGC } 4725 \text { mit Supernova } \\
\text { NGC } 4096 \text { mit Supernova } \\
\text { NGC } 4303 \text { mit Supernova } \\
\text { Centaurus A } \\
\text { Centaurus A }\end{array}$ \\
\hline $\begin{array}{l}95 \\
96 \\
97\end{array}$ & $\begin{array}{l}\text { Perseus A } \\
\text { Cygnus A } \\
\text { M87 (groß) }\end{array}$ \\
\hline 98 & M87 \\
\hline $\begin{array}{r}99 \\
100 \\
101 \\
102\end{array}$ & $\begin{array}{l}\text { M87 (klein) } \\
\text { M77 } \\
\text { NGC } 1566 \\
\text { M } 106\end{array}$ \\
\hline $\begin{array}{l}103 \\
104 \\
105,106 \\
107\end{array}$ & $\begin{array}{l}\text { M94 } \\
\text { NGC } 4151 \\
\text { NGC } 253 \\
\text { NGC2146 }\end{array}$ \\
\hline $\begin{array}{l}108 \\
109 \\
110\end{array}$ & $\begin{array}{l}\text { NGC } 2685 \\
\text { M51 und NGC } 5195 \\
\text { M51 }\end{array}$ \\
\hline $\begin{array}{l}111 \\
112 \\
113\end{array}$ & $\begin{array}{l}\text { M81 } \\
\text { M81 und M } 82 \\
\text { M81 und M } 82 \text { (Radiokarte) }\end{array}$ \\
\hline $\begin{array}{l}114 \\
115 \\
116 \\
117\end{array}$ & $\begin{array}{l}\text { M82 } \\
\text { M82 } \\
\text { NGC } 4631 \\
\text { NGC } 4631\end{array}$ \\
\hline $\begin{array}{l}118 \\
119\end{array}$ & $\begin{array}{l}\text { NGC } 5426 / 27 \\
\text { NGC } 2207 / / C 2163\end{array}$ \\
\hline 120 & NGC4567/68 \\
\hline
\end{tabular}

\section{Quelle}

Royal Observatory, Edinburg

D. H. Rogstad, California

Institute of Technology

Steven Strom, Kitt Peak Nationa

Observatory

Kitt Peak National Observatory

Kitt Peak National Observatory

Royal Observatory, Edinburgh

Kitt Peak National Observatory

Hale Observatories

Kitt Peak National Observatory

Cerro Tololo Inter-American

Observatory

Hale Observatories

United States Naval Observatory

United States Naval Observatory

K. Alexander Brownlee

Hale Observatories

Lick Observatory

Lick Hobservatory

Hale Observatories

Raymond J. Talbot, Jr., Reginald

$J$. Dufour, und Eric B. Jensen,

Rice University

Hale Observatories

Hale Observatories

Malcolm Smith und W.E. Harris,

Cerro Tololo Inter-American

Observatory

James Wray, McDonald

Observatory, University of Texas

Lick Observatory

Lick Observatory

Harvard College Observatory

James Wray, McDonald

Observatory, University of Texas

Kitt Peak National Observatory

Hale Observatories

Hale Observatories

Cerro Tololo Inter-American

Observatory

Kitt Peak National Observatory

Kitt Peak National Observatory

James Wray, McDonald

Observatory, University of Texas

Hale Observatories

Hale Observatories

S. T. Gottesman, University of

Florida, und L. Weliachen,

Madon Observatory

Hale Observatories

Hale Observatories

Kitt Peak National Observatory

James Wray, McDonald

Observatory, University of Texas

Lick Observatory

Vera Rubin, Cerro Tololo

Inter-American Observatory

Laird A. Thompson, Kitt Peak

National Observatory

\section{Photo Objekt}

122

Ab

123

124

125

126

127

128

129

130

131

132

133

134

134
135

136

Stephans Quintett

NGC 4038/39

NGC 2623

Object RG 33 No. 754

\section{NGC 2535/36}

NGC $7753 / 52$

NGC 5216/18

IC 1505

NGC 70

NGC 2275/2300

NGC 4438

NGC 5544/45

NGC 4676

Arp 273

NGC 5566/60/69

NGC 5221/22/26

Wagenrad Ring-Galaxie

Coma-Galaxienhaufen

Virgohaufen

Perseushaufen

Herkuleshaufen

Entfernter Haufen Abell 370

Shane-Wirtanen-Galaxienkarte

Quasar 3C273
Quelle

Hale Observatories

Hale Observatories

Hale Observatories

European Southern

Observatory

Halton Arp, Hale Observatories

Halton Arp, Hale Observatories

Halton Arp, Hale Observatories

Halton Arp, Hale Observatories

Halton Arp, Hale Observatories

Halton Arp, Hale Observatories

Halton Arp, Hale Observatories

Halton Arp, Hale Observatories

Halton Arp, Hale Observatories

Halton Arp, Hale Observatories

Halton Arp, Hale Observatories

Halton Arp, Hale Observatories Royal Observatory, Edinburgh

Laird A. Thompson, Kitt Peak

National Observatory

Kitt Peak National Observatory Kitt Peak National Observatory

Alan Dressler, Hale

Observatories

Barry Newell und W. J. Couch, Australian National University

M. Seldner, B. Siebers, E.J.

Groth, und P. J.E. Peebles

High Energy Astrophysics

Division, Harvard-Smithsonian

Center for Astrophysics
Abb. Titel

Der Platz der Sonne in den Spiralarmen unserer Galaxie

Die Lage der Magellanschen Wolken

Der Magellansche Strom

Die Lokale Gruppe

Galaxientypen

M51-NGC5195-Wechselwirkung

Rattenschwanz-Galaxien

Ringgalaxien

Nahe Galaxiengruppen

Ein endliches, aber unbegrenztes Strichland

Ein endliches, aber unbegrenztes Flachland

Raum-Zeit-Geometrie, die mit Hilfe von Lichtstrahlen dargestellt wird

Die Ausdehnung des Universums

Die Lichtkegel von Beobachtern in drei Galaxien

Darstellung der intergalaktischen Vergangenheit und

Zukunft mit Hilfe eines Lichtkegels

18 Darstellung des expandierenden, sich entwickelnden

Weltalls mit Hilfe der Rückblickzeit

19 Eine Urknall-Kosmologie mit drei Universen
Seite

$22-23$

42

$84-85$ 
D 
$\div$
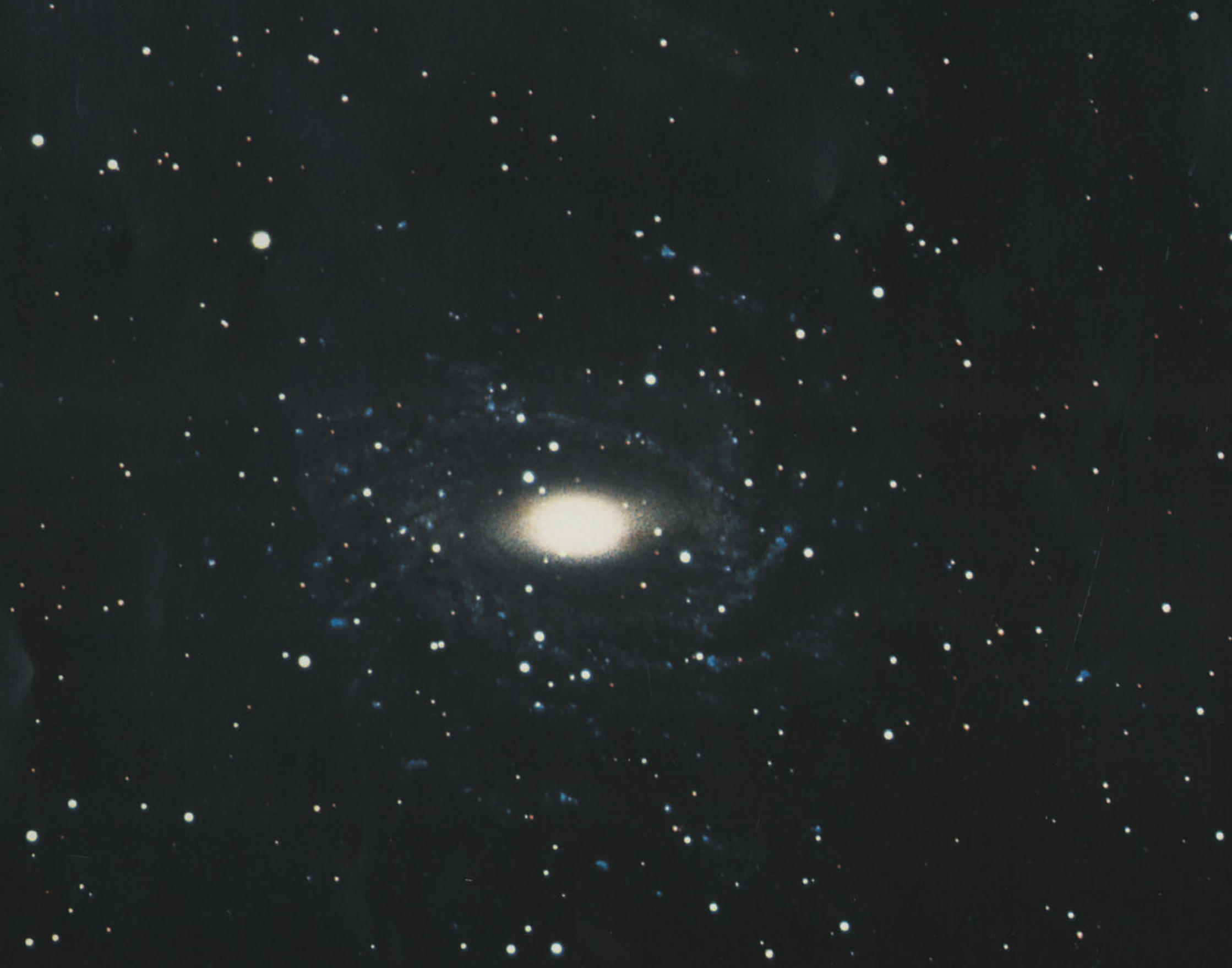


\section{G A L A X I E N}

Text und Fotoauswahl von Timothy Ferris

Mit einem Vorwort von A. Tammann

Springer Basel AG 
Frontispiz

Der Spiralnebel NGC6744 ist 300 Millionen Lichtjahre von der Erde entfernt; eine Schwarzweiß-Photographie dieser Galaxie erscheint auf Seite 109.

\section{CIP-Kurztitelaufnahme der Deutschen Bibliothek}

Galaxien / Text u. Fotoausw. von Timothy Ferris

Mit e. Vorw, von A Tammann. [Aus d. Amerikan.

übertr. von Anita Ehlers]. - Sonderausg. -

Basel ; Boston ; Stuttgart : Birkhäuser, 1983.

Einheitssacht.: Galaxies

ISBN 978-3-7643-1488-0

NE: Ferris, Timothy [Bearb.]; EST

Die vorliegende Publikation ist urheberrechtlich geschützt.

Alle Rechte vorbehalten. Kein Teil dieses Buches darf ohne schriftliche Genehmigung des Verlages in irgendeiner Form - durch Fotokopie, Mikrofilm oder andere Verfahren - reproduziert oder in eine für Maschinen, insbesondere Datenverarbeitungsanlagen, verwendbare Sprache übertragen werden. Auch die Rechte der Wiedergabe durch Vortrag, Funk und Fernsehen sind vorbehalten.

\section{(C) 1983 Springer Basel AG}

Ursprünglich erschienen bei Birkhäuser Verlag Basel 1983

ISBN 978-3-7643-1488-0

ISBN 978-3-0348-6774-0 (eBook)

\section{Über den Autor}

Timothy Ferris, 37, ist Associate Professor für Englisch am Brooklyn College der City Universität von New York. T. Ferris ist durch seine Veröffentlichungen über Astronomie und Weltraumfahrt bekannt geworden. Sein erstes populäres Buch،The Red Limit: The Search for the Edge of the Universe, erhielt 1978 den Preis des American Institute of Physics. Für seine Berichterstattung in 'Rolling Stone, über die Viking-Landung auf dem Mars wurde er mit dem ersten Preis der Aviation/Space Writers Association ausgezeichnet. Ferris hat außerdem maßgeblich an der Konzeption und Produktion jenes Bild-/Tonmediums mitgewirkt, das als Botschaft der Menschheit an außerirdische Zivilisationen 1977 an Bord der Voyager-Raumsonden auf eine interstellare Reise ging. Dieses Unternehmen ist in dem Buch ‘Signal der Erde` näher beschrieben, an dem Timothy Ferris als Mitverfasser beteiligt ist.

DOI 10.1007/978-3-0348-6774-0 


\section{Vorwort}

Es gibt kaum eine Wissenschaft, in der eine solche Kluft zwischen ihrem Bild in der Öffentlichkeit und ihren wirklichen Aufgaben und Zielen besteht, wie die Astronomie. Während weithin noch angenommen wird, die Astronomie begnüge sich damit, Sternbilder und neuentdeckte Sterne zu benennen, oder sie habe etwas mit den spekulativen Sumpfblüten der Astrologie zu tun, bemüht sich die astronomische Forschung heute um ein physikalisches Verständnis des Verhaltens der Materie in kosmischen Räumen und über sehr lange Zeiten: um die Entstehung, um den Aufbau und um das Ende von Sternen, von Sonnensystemen, von Galaxien und des Universums als Ganzem.

Die astronomische Forschung und die Öffentlichkeit einander näher zu bringen, ist eine außerordentlich wichtige Aufgabe, da die Astronomie in allen Kulturstaaten aus öffentlichen Mitteln unterstützt wird. Dementsprechend hat die Öffentlichkeit einen Anspruch auf einen Rechenschaftsbericht, dies um so mehr, weil gewisse astronomische Erkenntnisse praktische Bedeutung haben können, und weil sie in starkem Maße das Weltbild des modernen Menschen beeinflussen.

Die Schwierigkeit der Kommunikation zwischen Wissenschaftlern und der Öffentlichkeit liegt darin, daß jene eine weitgehend mathematische Fachsprache entwickelt haben, die zwar außerordentlich rationell und klar ist, die aber dem Nichtspezialisten ohne großen Zeitaufwand nicht mehr zugänglich ist. Es bedarf daher spezieller Mittler, die einerseits die Fachsprache in eine allgemein verständliche Sprache übersetzen und andererseits aus der Fülle von vorliegenden Einzelergebnissen die wichtigsten Grunderkenntnisse herauskristallisieren.

Für diese Mittlerrolle kommen vor allem zwei Berufsgruppen in Frage: die Wissenschaftler selber, die ein reich beladenes
Fachwissen haben, die aber mit ihrer Fachsprache mehr oder weniger behaftet sind, und diejenigen, die die Alltagssprache in professioneller Weise beherrschen und benützen, wie etwa Schriftsteller und Journalisten, denen das unerläßliche wissenschaftliche Verständnis aber meistens fehlt.

Das Reizvolle an dem vorliegenden Buche ist, daß sein Autor, Timothy Ferris, als Englisch-Professor, Schriftsteller und Journalist die Sprache in wahrhaft professioneller, ja oft poetischer Weise beherrscht, und daß er die Aufgabe, sich in die moderne Astronomie mit ihren Ergebnissen und ihrer Denkweise einzuarbeiten, außerordentlich ernst genommen hat. Auf weiten Reisen und während oft wochenlangen Aufenthalten hat er an den großen Observatorien geweilt und mit unzähligen führenden Wissenschaftlern engen Kontakt aufgenommen. Er kennt und versteht nicht nur deren Ergebnisse, sondern er hat eine Gesamtschau der Astronomie gewonnen, um die ihn viele Spezialisten beneiden müssen.

Der Text, dessen Originaltät und Anschaulichkeit auch in der hier vorliegenden deutschen Übersetzung voll zum Tragen kommen, ist nur die eine Säule dieses Buches. Die Bilder liefern die andere. Der Autor hat mit großer Sorgfalt die schönsten astronomischen Photographien zusammengetragen, und er versteht es, als nicht nur profunder Kenner der Materie deren wissenschaftliche Aussagekraft zu erläutern, sondern auch als Künstler deren oft großartige Schönheit zu vermitteln.

Ich glaube, daß der interessierte Leser, der nicht die Einzelergebnisse der Astronomie kennenlernen will, sondern der eine umfassende Darstellung des Wesens der modernen Astronomie sucht, zu keinem besseren und im wahren Sinne des Wortes zu keinem schöneren Buch greifen kann.

Basel, 1983

A. $T$. 


\section{Dank}

Dies sind die Namen einiger, die mich freundlicherweise bei Galaxien unterstützt haben. Da keiner von innen das Buch in seiner endgültigen Form gesehen hat, trägt der Verfasser allein die Verantwortung für Irrtümer oder Unzulänglichkeiten.

\section{Information und Photographien}

Halton Arp, Elly M. Berkhuijsen, Richard Berry, K. Alexander Brownlee, Lloyd Carter, S. Chandrasakhar, Mark R. Chartrand III, J. N. Clarke, James Cornell, A. G. de Bruyn, Terry Dickinson, Alan Dressler, Reginald Dufour, Vince Ford, Ken Franklin, Paul Gorenstein, J. Richard Gott III, Stephen T. Gottesman, John Graham, Edward J. Groth, B. W. Hadley, W. E. Harris, Eric B. Jensen, T. D. Kinman, Martha Liller, David Malin, Dennis Meredith, Simon Mitton, Richard Müller, Barry Newell, Rene Racine, Connie Rodriguez, D. H. Rogstad, Paul Routly, Ronald E. Royer, Vera Rubin, Allan Sandage, Jan Schafer, Malcolm Smith, Stephen Strom, Laird A. Thompson, Alar Toomre, Sindey van den Bergh, J. M. van der Hulst, Gerard de Vaucouleurs, Richard M. West, Fujiko Worrell, James D. Wray, Anglo-Australian Observatory, Astrophoto Laboratory, Australian National University, Brooklyn College der City Unversity of New York, California Institute of Technology, Cambridge University, Carnegie Institution of Washington, Cerro Tololo Inter-American Observatory, Dominion Astrophysical Observatory, European Southern Observatory, Griffith Observatory, Hale Observatories, Harvard College Observatory, Hayden Planetarium, Kitt Peak National Observatory, Lawrence Berkeley Laboratory of the University of California, Lick Observatory, Los Angeles Public Library, Max-Planck-Institut für Radioastronomie, McDonald Observatory, McMaster University, Mt. Stromlo and Siding Springs Observatories, New York Public Library, Princeton University, Rice University, Royal Observatory Eding- burgh, Smithsonian Astrophysical Observatory, United States Naval Observatory, United States Naval Research Laboratory, Université de Montréal, University of Chicago, University of Florida, University of Minnesota, University of Texas at Austin, University of Toronto, Westerbork Radio Observatory.

\section{Redaktionelle und fachliche Beratung}

Timothy Crouse, Alan Dressler, J. Richard Gott III, Lynda Obst, Dennis Overbye, R. Bruce Partridge, Thomas M. Powers, Stephen Strom, Gerard de Vaucouleurs.

\section{Unterstützung und Ermutigung}

Jon Beckmann, Monica Brown, Jean B. Ferris, Wendy Goldwyn, Kathy Lowry, Lynda Obst, Bruce Porter, Thomas M. Powers, Delfina Rattazzi, Lisa Robinson, Allan Sandage, Alex Shoumatoff, Erica Spellman, Caroly Zecca.

\section{Wissenschaftliche Beratung}

Eileen Casey, Eustice Clarke, Juan de Jesus, Robert Ginsberg, Sandra Kitt, Judy Mitko, Terry Tammadge.

\section{Illustrationen}

Sarah Landry

\section{Widmung}

Den Astronomen überall 


\section{Inhalt}

\section{Einleitung}

Wo sind wir? 10

Die Entdeckung der Galaxien 11

Zu den Photographien 14

Galaxien und menschliches Denken

15

\section{I}

Die Milchstraße: Ein Spiralnebel von innen gesehen Eine Reise zum Mittelpunkt der Milchstraße 18

Die Milchstraße 20

Die Sonne 20

Sterne und interstellarer Raum 24

Sterngeburten 28

Der Orionnebel 28

Der Adlernebel 32

Der Rosettanebel 34

Der Eta Carinae-Nebel 39

Die Trifid- und Lagunennebel 43

Offene Sternhaufen 46

Kugelsternhaufen 50

Sternentod 53

Planetarische Nebel 55

Ausbrechende und explodierende Sterne 56

Schwarze Löcher 60

Die Struktur des Milchstraßensystems

II

Die Lokale Gruppe von Galaxien

Eine Reise aus unserer Galaxis hinaus 68

Die Lokale Gruppe 70

Die Magellanschen Wolken 70

Der Andromedanebel 76

Die Galaxie M33 82

Die Bildhauer-Zwerggalaxie 86

III

Form und Vielfalt der Galaxien

Eine Reise durch intergalaktischen Raum 88

Form und Vielfalt von Galaxien 90

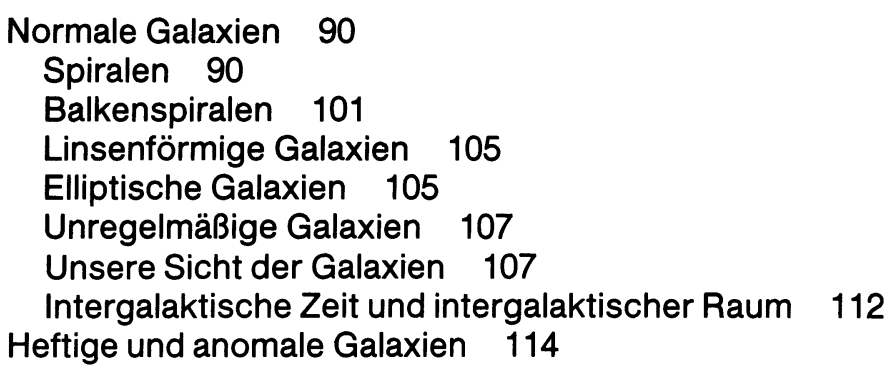

Eine Reise zwischen wechselwirkenden Galaxien 128

Galaxien in Wechselwirkung 130

Das System M51-NGC5195 130

Das System M81-M82 135

Die Galaxie NGC4631 139

Unterschiede in der Wechselwirkung 141

'Rattenschwanz'-Galaxien 142

Ringgalaxien 144

V

\section{Galaxienhaufen}

Eine Reise durch den Lokalen Superhaufen 150

Galaxienhaufen 152

Form und Vielfalt von Haufen und Superhaufen 152

Die Virgo- und Comahaufen 154

Herkules- und Perseushaufen 156

\section{VI}

Galaxien und das Weltall

Eine Reise ans Ende des Weltalls 160

Galaxien und das Weltall . 162

Geometrien von Raum und Zeit 162

Die Ausdehnung des Weltalls 166

Rückblickzeit 169

Kosmologische Modelle 175

Bildquellen 178

Ausgewählte Bibliographie 180

Glossar 182

Register 187 